OPEN ACCESS

Edited by:

Mihajlo Jakovljevic,

Hosei University, Japan

Reviewed by:

Pengfei Zhang,

Sichuan University, China

Nan Zhang,

West China Hospital, China

${ }^{*}$ Correspondence:

Yizhun Zhu

yzzhu@must.edu.mo

tThese authors have contributed equally to this work

Specialty section

This article was submitted to

Health Economics,

a section of the journal

Frontiers in Public Health

Received: 24 August 2021 Accepted: 06 October 2021 Published: 29 October 2021

Citation:

Liu X, Lang Y, Liao Y and Zhu Y (2021) Atezolizumab Plus Chemotherapy vs.

Chemotherapy in Advanced or

Metastatic Triple-Negative Breast

Cancer: A Cost-Effectiveness

Analysis.

Front. Public Health 9:756899.

doi: 10.3389/fpubh.2021.756899

\section{Atezolizumab Plus Chemotherapy vs. Chemotherapy in Advanced or Metastatic Triple-Negative Breast Cancer: A Cost-Effectiveness Analysis}

\author{
Xiaoyan Liu ${ }^{1,2 \dagger}$, Yitian Lang ${ }^{1 \dagger}$, Yahui Liao ${ }^{1}$ and Yizhun $\mathrm{Zhu}^{2 *}$ \\ ${ }^{1}$ Department of Pharmacy, Huangpu Branch, Shanghai Ninth People's Hospital, Shanghai Jiao Tong University School of \\ Medicine, Shanghai, China, ${ }^{2}$ State Key Laboratory of Quality Research in Chinese Medicine, School of Pharmacy, Macau \\ University of Science and Technology, Taipa, Macau SAR, China
}

Purpose: The IMpassion130 trial demonstrated the efficacy of adding atezolizumab to paclitaxel for advanced or metastatic triple-negative breast cancer (TNBC). The current study evaluated the cost-effectiveness of adding atezolizumab to nab-paclitaxel for TNBC from the perspective of Chinese health sector.

Methods: A partitioned survival model was implemented for patients with TNBC. The survival data were derived from IMpassion130 trial. Direct costs and utility values were collected from the Chinese Drug Bidding Database and published literatures. The primary analysis outcomes were quality-adjusted life-years (QALYS) and incremental cost-effectiveness ratios (ICERs). Sensitivity analyses were performed to observe model stability.

Results: In the base-case analysis, the ICER of atezolizumab plus nab-paclitaxel vs. nab-paclitaxel is respectively, \$176,056/QALY, \$118,146/QALY, and \$323,077/QALY in the ITT, PD-L1(+) and PD-L1(-) group.

Conclusion: Adding atezolizumab to nab-paclitaxel could improve survival time significantly in the PD-L1-positive group, but it is not a cost-effective strategy compared to nab-paclitaxel monotherapy for Chinese patients with advanced or metastatic triple-negative breast cancer in the current economic context of China.

Keywords: atezolizumab, cost-effectiveness, partitioned survival model, triple-negative breast cancer, the perspective of China

\section{INTRODUCTION}

Breast cancer is the most common female malignant cancer and accounts for around one-tenth of all new diagnosed cancers worldwide (1). The incidence has been generally rising over the last 50 years with rapid increases observed particularly in developing countries $(2,3)$. Molecular markers depend primarily on the expression of relevant receptors in breast cancer, including estrogen receptor (ER), progesterone receptor (PR) and human epidermal growth factor receptor 2 (HER2). Various expressions of these receptors classified into major subtypes include luminal A, luminal B, 
HER2-overexpressing and triple-negative breast cancer (TNBC) (4-6). TNBC, an intrinsic subtype of breast cancer, is defined as a tumor that does not express the ER, PR, or HER2 and accounted for $15-20 \%$ of all breast cancer cases. As this type of cancer cell does not respond to hormone therapy or targeted drugs, it brings a big challenge to clinical treatment (7). Therefore, chemotherapy, especially chemotherapy with paclitaxel as the main component, is still the first choice for clinical therapeutic regimen $(8,9)$. However, there are still many new regimens being explored. In recent years, cancer immunotherapy has been applied to various cancers when no long-term responses were observed with cytotoxic chemotherapy. All immunotherapies have a similar mechanism of action that forces the body's own immune system to eliminate cancer cells. Programmed deathprotein $1(\mathrm{PD}-1)$ /programmed death ligand-1(PD-L1) pathway is one mechanism for tumor cells to avoid anti-tumor immune response. The reason is that when the PD-L1 of cancer cell binds to the $\mathrm{PD}-1$ receptor of $\mathrm{T}$ cells, the expansion and activity of cytotoxic $\mathrm{T}$ cells are suppressed (10). However, the PD-1/PD-L1 inhibitors can block the pathway. At present, many clinical trials have been conducted for TNBC patients using PD-1/PD-L1 inhibitors (11). KEYNOTE-355 trial show that the progression-free survival (PFS) was 9.7 months with pembrolizumab plus chemotherapy and 5.6 months with placebo plus chemotherapy in CPS of 10 or more TNBC patients at the second interim analysis (12). IMpassion130 trial similarly explored the efficacy of immune checkpoint inhibitors (ICIs) adding to first-line treatment. Among TNBC patients with PDL1(+), median progression-free survival was 7.5 months with atezolizumab plus chemotherapy, as compared with 5.0 months with placebo plus chemotherapy (13). And the overall survival was 25.0 months with atezolizumab plus chemotherapy and 18.0 months with placebo plus chemotherapy (14). It indicated that PD-1/PD-L1 inhibitors bring significant clinical benefits for $\mathrm{PFS} / \mathrm{OS}$ in the PD-L1(+) TNBC patients. Therefore, some PD1/PD-L1 inhibitors such as atezolizumab have been approved by FDA in 2019 (11). At present, atezolizumab is available in China, its price and economic burden are uncertain for Chinese patients. As far as we know, some studies have assessed the cost-effectiveness of atezolizumab for TNBC from the US payer perspective. However, no research has been conducted to analyze the potential economic burden of the therapeutic regimen from the Chinese perspective. We evaluated the costeffectiveness of atezolizumab plus nab-paclitaxel vs. placebo plus paclitaxel in TNBC patients from the perspective of Chinese health sector.

\section{MATERIALS AND METHODS}

\section{Model Structure}

In this study, we used a partitioned survival model to simulate the disease survival states of TNBC patients beyond the follow-up time of clinical trial and make a cost-effectiveness analysis. The target population of the study was kept with that of the IMpassion130 trial, who were aged 18 years or older and had been confirmed histologically documented, unresectable, locally advanced or metastatic TNBC. The patients of this study receive one of two interventions until disease progression: (1) chemotherapy (nab-paclitaxel); (2) atezolizumab plus chemotherapy. When suffered disease progressed, it is assumed that initial regimen became invalid and the current therapy regimen was terminated. And then, $53.7 \%$ of patients in the atezolizumab-nab-paclitaxel group and $60.3 \%$ in the placebo-nab-paclitaxel group receive subsequent best supportive anticancer regimens.

The partitioned survival model was composed of three mutually exclusive health states, which are respectively progression-free (PF) survival, progressed disease (PD) and death. Figure 1 shows the tree diagram and bubble diagram. The TNBC patients entered the model in PFS state primarily and then could move to the PD or death states based on survival data. The duration of the model cycle was 28 days, which was consistent with the treatment protocol in IMpassion130. Extrapolating short-term and limited results to reflect long-term prognosis can fully understand the outcome of the disease. So, the time horizon of 10 years was essential to ensure that TNBC patients fully entered the terminal state.

\section{Clinical Data}

The available observational time of IMpassion 130 trial was around 42 months for OS and PFS. To predict the survival outcomes over a 10-year horizon, a method that extrapolated over the follow-up time was used based on algorithms proposed by Guyot et al. (15). The tool of digitizing the OS and PFS Kaplan-Meier curves for interventions is Engauge Digitizer (version 12.1, https:/github.com/markummitchell/ engauge-digitizer/releases). The generated simulated individual patient level data (IPD) were applied to fit following parametric distributions: Weibull, Gompertz, exponential, log-normal, and log-logistic distributions. The best fitted distribution of IPD is selected based on Akaike information criterion (AIC) value (16).

\section{Costs and Utilities}

This analysis was conducted from the perspective of the Chinese health sector. Only direct medical costs were considered, including costs related to drugs, management of adverse events and palliative care. The drug dose is consistent with that of the IMpassion130. In the chemotherapy regimen, nab-paclitaxel was used at a dose of $100 \mathrm{mg}$ body surface area (BSA) per square meter on days 1,8 , and 15 of each model cycle. In atezolizumab plus chemotherapy regimen, atezolizumab was administered at a dose of $840 \mathrm{mg}$ on days 1 and 15 of each model cycle and nab-paclitaxel is administered in keeping with the above-mentioned chemotherapy regimen. The mean BSA of Chinese patients is $1.72 \mathrm{~m}^{2}(17)$. The prices of nab-paclitaxel and atezolizumab in China were acquired from drug acquisition costs in local charge database (18). Costs related to terminal care and subsequent best supportive care (BSC) were derived from published literatures $(19,20)$. The IMpassion 130 trial shared data about incidences of adverse events. And only the costs related to managing grade 3 and more AEs were included. Grade 1 and 2 


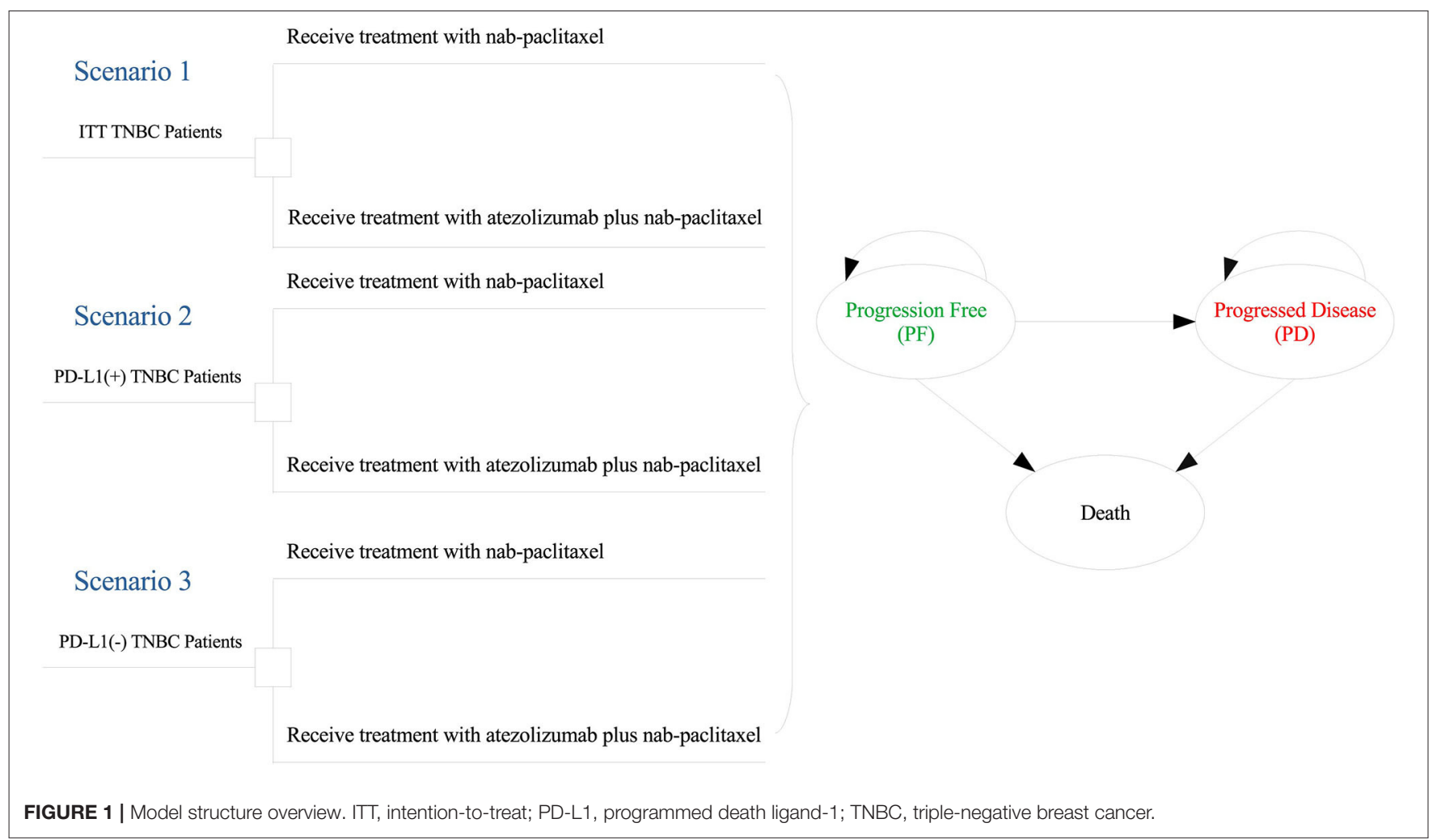

AEs could be well-managed, the costs of management were not considered. The costs of managing grade 3-5 AEs were sourced from previously published economic studies $(21,22)$. All costs reported for years prior to 2020 were updated to 2020 US dollars (US\$) using the health care services component of Chinese Consumer Price Index. More details about costs are summarized in Table 1.

Every health state was assigned a health utility value in this partitioned survival model. Since the IMpassion130 trial lacked research about the quality-of-life data of TNBC patients, other robust data is extremely important. As the quality of life is associated with progressive stage, the utility values in metastatic/advanced breast cancer and TNBC were assumed to be consistent. The utility estimates for PF state and PD state were assumed to be 0.843 and 0.60 based on data collected from studies about locally advanced and metastatic breast cancer (23). In addition, the values of disutility due to grade 3-5 main AEs should be considered, the values were derived from relevant economic studies (24).

\section{Analyses}

Our analyses covered three scenarios (Figure 1): intentionto-treat (ITT) TNBC patients (Scenario 1), PD-L1-positive (expression on tumor-infiltrating immune cells $\geq 1 \%$ ) TNBC patients (Scenario 2) and PD-L1-negative (expression on tumorinfiltrating immune cells $<1 \%$ ) TNBC patients (Scenario 3). In each scenario, the treatment regimens received by the patients does not change and only the survival data of patients are different among all scenarios.
In the base-case analysis, we used incremental costeffectiveness ratios (ICERs) to evaluate the incremental cost per additional quality-adjusted life-year (QALY) gained between atezolizumab plus nab-paclitaxel and nab-paclitaxel regimen. A $3 \%$ annual discount rate was applied for all costs and QALYs. If the ICER of atezolizumab plus nab-paclitaxel compared with nab-paclitaxel is below the willingness-to-pay (WTP) threshold, atezolizumab plus nab-paclitaxel regimen is considered "costeffective." The WTP threshold of three times the per capita gross domestic product (GDP) was recommended and is calculated to be $\$ 31,316$ in China $(25,26)$.

In order to assess the robustness of our results and identify the variables that have considerable impacts on the analysis results, we conducted one-way and probabilistic sensitivity analyses (PSA) for model input parameters. In one-way sensitivity analyses, the range of every input parameter was assumed a variation by $\pm 25 \%$. The range of discount rate is between 0 and $8 \%$. In addition, the PSA was conducted by a Monte Carlo simulation of 1,000 iterations. All input parameters were sampled simultaneously based on specific probability distributions. Health utilities, disutility values and probabilities of adverse events were sampled from Beta distribution, and the costs were sampled from Gamma distribution (27). The PSA outcomes are presented as cost-effectiveness acceptability curve (CEAC) to illustrate the likelihood that atezolizumab plus nabpaclitaxel regimen was cost-effective at specific WTP threshold. The partitioned survival model and cost-effectiveness analysis model were created and programmed in $\mathrm{R}$ (version 4.0.5, http:// www.r-project.org). 
TABLE 1 | Input parameters of the model.

\begin{tabular}{|c|c|c|c|c|}
\hline Parameters & Expected value & Range & Distribution & References \\
\hline \multicolumn{5}{|l|}{ Clinical inputs } \\
\hline \multicolumn{5}{|l|}{ ITT TNBC patients } \\
\hline OS: Atezolizumab + nab- paclitaxel & $\begin{array}{l}\text { shape: } 1.6892 \\
\text { scale: } 22.1345\end{array}$ & NA & Log-logistic & (14) \\
\hline OS: nab-paclitaxel & $\begin{array}{l}\text { shape: } 1.3805 \\
\text { scale: } 26.3036\end{array}$ & NA & Weibull & (14) \\
\hline \multicolumn{5}{|c|}{ TNBC patients with PD-L1-positive status } \\
\hline PFS: Atezolizumab + nab-paclitaxel & $\begin{array}{l}\text { meanlog: } 2.0798 \\
\text { sdlog: } 1.0586\end{array}$ & NA & Lognormal & (14) \\
\hline OS: nab-paclitaxel & $\begin{array}{l}\text { shape: } 1.301 \\
\text { scale: } 25.56\end{array}$ & NA & Weibull & (14) \\
\hline \multicolumn{5}{|c|}{ TNBC patients with PD-L1-negative status } \\
\hline PFS: Atezolizumab + nab-paclitaxel & $\begin{array}{l}\text { shape: } 1.934 \\
\text { scale: } 6.657\end{array}$ & NA & Log-logistic & (14) \\
\hline OS: Atezolizumab + nab- paclitaxel & $\begin{array}{l}\text { shape: } 1.815 \\
\text { scale: } 20.045\end{array}$ & NA & Log-logistic & (14) \\
\hline PFS: nab-paclitaxel & $\begin{array}{l}\text { shape: } 1.8035 \\
\text { scale: } 6.2604\end{array}$ & NA & Log-logistic & (14) \\
\hline OS: nab-paclitaxel & $\begin{array}{l}\text { shape: } 1.456 \\
\text { scale: } 26.4987\end{array}$ & NA & Weibull & (14) \\
\hline Fatigue & $\$ 131.78$ & $98.84-164.73$ & Gamma & (22) \\
\hline Anemia & $\$ 607.06$ & $455.30-758.83$ & Gamma & (22) \\
\hline Neutropenia & $\$ 526.90$ & $395.18-658.63$ & Gamma & (22) \\
\hline Neutrophil count decreased & $\$ 104.95$ & $78.71-131.19$ & Gamma & (21) \\
\hline \multicolumn{5}{|l|}{ Utility estimates } \\
\hline Progression free survival & 0.843 & $0.632-1$ & Beta & (23) \\
\hline Progressed disease & 0.60 & $0.45-0.75$ & Beta & (23) \\
\hline \multicolumn{5}{|l|}{ Disutility estimates } \\
\hline Utility reduction due to fatigue & -0.029 & -0.022 to -0.036 & Beta & (24) \\
\hline Utility reduction due to anemia & -0.029 & -0.022 to -0.036 & Beta & (24) \\
\hline Utility reduction due to neutropenia & -0.012 & -0.050 to -0.083 & Beta & (24) \\
\hline \multicolumn{5}{|c|}{ Probability of main grade 3-5 adverse events in atezolizumab plus nab-paclitaxel arm } \\
\hline Fatigue & $3.8 \%$ & $2.9-4.8 \%$ & Beta & (14) \\
\hline Anemia & $3.1 \%$ & $2.3-3.9 \%$ & Beta & (14) \\
\hline Neutropenia & $8.4 \%$ & $6.3-10.5 \%$ & Beta & (14) \\
\hline Neutrophil count decreased & $4.9 \%$ & $3.7-6.1 \%$ & Beta & (14) \\
\hline
\end{tabular}


TABLE 1 | Continued

\begin{tabular}{|c|c|c|c|c|}
\hline Parameters & Expected value & Range & Distribution & References \\
\hline \multicolumn{5}{|c|}{ Probability of main grade 3-5 adverse events in nab-paclitaxel arm } \\
\hline Fatigue & $3.4 \%$ & $2.6-4.3 \%$ & Beta & $(14)$ \\
\hline Anemia & $3 \%$ & $2.3-3.8 \%$ & Beta & $(14)$ \\
\hline Neutropenia & $8.2 \%$ & $6.2-10.3 \%$ & Beta & $(14)$ \\
\hline Neutrophil count decreased & $3.7 \%$ & $2.8-4.6 \%$ & Beta & $(14)$ \\
\hline \multicolumn{5}{|l|}{ Other parameters } \\
\hline Body surface area & 1.72 & $1.52-1.92$ & Normal & (17) \\
\hline
\end{tabular}

All costs sourced from China in this study were converted into US dollars (\$1=RMB 6.8974 in 2020).

BSC, best supportive care; ITT, intention-to-treat; PD-L1, programmed death ligand-1; TNBC, triple-negative breast cancer; PFS, Progression-free survival; OS, Overall survival.

\section{RESULTS}

\section{Validity of the Fitted Parametric Survival Function}

The validation by comparing the observational and predicted curves is shown in Figure 2. The distribution of projected curve could be seen in Table 1.

\section{Base-Case Analysis}

All base-case results were summarized in Table 2 .

\section{Scenario 1}

In the group of intention-to-treat patients, the base-case analysis results showed that life-year (LY), QALY and cost were 2.606, 1.724 , and $\$ 83,700$ in the atezolizumab plus nab-paclitaxel regimen. $1.87,1.297$, and $\$ 8,524$ in the nab-paclitaxel regimen. Compared with nab-paclitaxel regimen, patients received atezolizumab plus nab-paclitaxel increased the cost by $\$ 75,176$ with the augments of 0.736 LYs and 0.427 QALYs. The average cost-effectiveness ratios of atezolizumab plus nab-paclitaxel are $\$ 32,118 / \mathrm{LY}$ and $\$ 48,550 / \mathrm{QALY}$. And that of nab-paclitaxel regimen is $\$ 4,558 /$ LYs and $\$ 6,572 /$ QALYs. The ICER of atezolizumab plus nab-paclitaxel compared with nab-paclitaxel is $\$ 102,141 / \mathrm{LY}$ and \$176,056/QALY.

\section{Scenario 2}

In the PD-L1 (+) group, the base-case analysis results showed that LY, QALY and cost were 3.101, 2.035, and $\$ 99,688$ in the atezolizumab plus nab-paclitaxel regimen. 1.836 LY, 1.259 QALYs and $\$ 8,007$ in the nab-paclitaxel regimen. Patients receiving atezolizumab plus nab-paclitaxel had a $\$ 91,681$ increase in cost with a rise of 1.265 LYs and 0.776 QALYs compared with nab-paclitaxel alone. The mean cost-effectiveness ratios for atezolizumab plus nabpaclitaxel are \$32,147/LY and \$48,987/QALY. And nab-paclitaxel regimen is $\$ 4,361 / \mathrm{LYs}$ and $\$ 6,360 / \mathrm{Q} A L Y$ s. The ICER for atezolizumab plus nab-paclitaxel vs. nab-paclitaxel is $\$ 72,475 / \mathrm{LY}$ and $\$ 118,146 /$ QALY.

\section{Scenario 3}

In the group of PD-L1(-) patients, LY, QALY and cost were $2.316,1.548$, and $\$ 76,411$ in the atezolizumab plus nab-paclitaxel regimen. 1.907 LY, 1.34 QALYs and \$9,211 in the nab-paclitaxel regimen. In comparison with patients receiving only nabpaclitaxel, atezolizumab plus nab-paclitaxel led to an additional $\$ 67,200$ in cost with an increase of 0.409 LYs and 0.208 QALYs. The mean cost-effectiveness ratios of atezolizumab plus nab-paclitaxel are $\$ 32,993 / \mathrm{LY}$ and $\$ 49,361 / \mathrm{QALY}$. Nabpaclitaxel regimen is $\$ 4,830 / \mathrm{LYs}$ and $\$ 6,874 / \mathrm{QALYs}$. The ICER for atezolizumab plus nab-paclitaxel vs. nab-paclitaxel is \$164,303/LY and \$323,077/QALY.

\section{One-Way Sensitivity Analysis}

The one-way sensitivity analyses are shown in the tornado diagrams. In all scenarios, the price of atezolizumab, utility of progressed disease and discount rate have substantial influence on the ICERs between atezolizumab plus nab-paclitaxel and nab-paclitaxel. The range for the one-way sensitivity analysis was from \$132,732/QALY to \$218,788/QALY in the ITT group (scenario 1, Supplementary Figure 1A). The range of PD-L1-positive group was between \$89,792/QALY and $\$ 146,514 /$ QALY (scenario 2, Figure 3). PD-L1-negative group ranged between $\$ 241,981 /$ QALY and \$428,361/QALY (scenario 3, Supplemental Figure 1B).

\section{Probabilistic Sensitivity Analysis}

One thousand iterations were conducted to evaluate all model parameters sampling from probability distributions simultaneously. At the threshold of \$31,316/QALY, the costeffectiveness acceptable curve (CEAC) showed that a $0 \%$ likelihood of atezolizumab plus nab-paclitaxel regimen being cost-effective in various groups (ITT, PD-L1-positive and PD-L1negative patients). In addition, the simulations about adjusting the cost of atezolizumab at 75,50 , and $25 \%$ price were also conducted. Notably, the CEAC (Figure 4) indicated that the chance of atezolizumab plus nab-paclitaxel regimen being costeffective were $0,1.3$, and $38.7 \%$ at 75,50 , and $25 \%$ price with a WTP threshold of $\$ 31,316 / \mathrm{QALY}$ in the PD-L1-positive patients. And the CEAC of ITT group (Supplementary Figure 2A) showed 0,0 , and $8.5 \%$ likelihood at 75, 50, and 25\% price with a WTP threshold of $\$ 31,316 /$ QALY. Likewise, nearly $0 \%$ probability at 75,50 , and $25 \%$ price of atezolizumab in the PD-L1-negative group (Supplementary Figure 2B). 


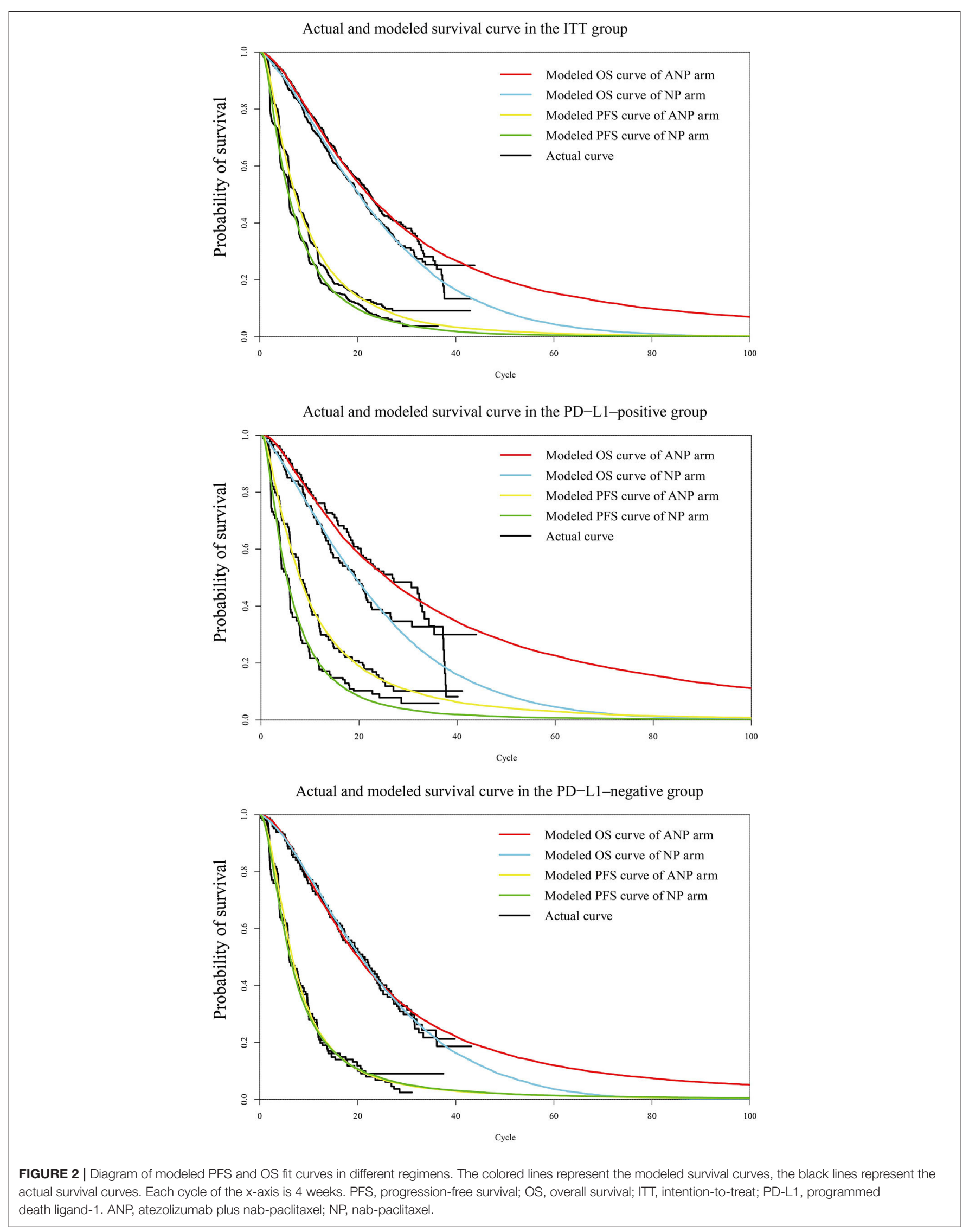


TABLE 2 | Results of the base-case analysis.

\begin{tabular}{|c|c|c|c|c|c|c|c|c|}
\hline Scenario & Regimen & $\begin{array}{l}\text { Total cost } \\
\text { (USD) }\end{array}$ & LYs & QALYs & $\begin{array}{c}\text { CER } \\
\text { (USD/LY) }\end{array}$ & $\begin{array}{c}\text { CER } \\
\text { (USD/QALY) }\end{array}$ & $\begin{array}{c}\text { ICER } \\
\text { (USD/LY) }\end{array}$ & $\begin{array}{c}\text { ICER } \\
\text { (USD/QALY) }\end{array}$ \\
\hline \multirow[t]{2}{*}{ ITT patients } & Nab-paclitaxel & 8,524 & 1.87 & 1.297 & 4,558 & 6,572 & & \\
\hline & Atezolizumab + nab-paclitaxel & 83,700 & 2.606 & 1.724 & 32,118 & 48,550 & 102,141 & 176,056 \\
\hline \multirow[t]{2}{*}{ PD-L1(+) patients } & Nab-paclitaxel & 8,007 & 1.836 & 1.259 & 4,361 & 6,360 & & \\
\hline & Atezolizumab + nab-paclitaxel & 99,688 & 3.101 & 2.035 & 32,147 & 48,987 & 72,475 & 118,146 \\
\hline \multirow[t]{2}{*}{ PD-L1(-) patients } & Nab-paclitaxel & 9,211 & 1.907 & 1.34 & 4,830 & 6,874 & & \\
\hline & Atezolizumab + nab-paclitaxel & 76,411 & 2.316 & 1.548 & 32,993 & 49,361 & 164,303 & 323,077 \\
\hline
\end{tabular}

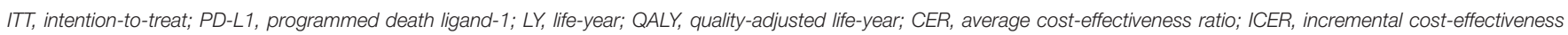
ratio; USD, United States dollar.

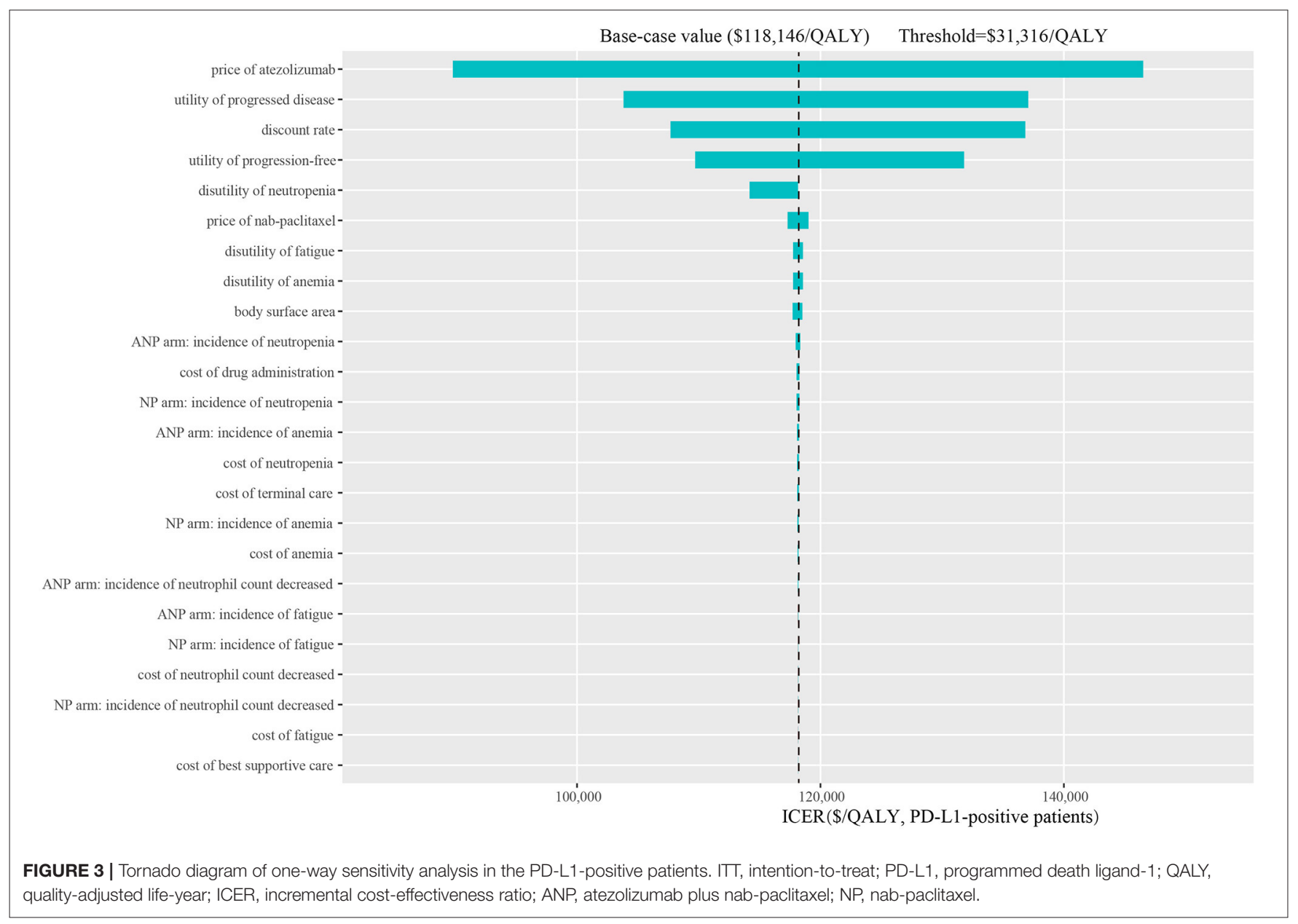

\section{DISCUSSION}

Since TNBC patients express none of these three receptors (ER, PR, and HER2) exhibit a worse prognosis, making anti-tumor immune system re-identify tumor cells through inhibiting the programmed cell death 1 pathway became a solution that can be tried (28). Atezolizumab as a PD-L1 inhibitor was reported to have better clinical benefit for TNBC patients in IMpassion130 trial, which brought hope to both breast cancer patients and clinicians. And then, atezolizumab was approved in February
2020 for the treatment of small cell lung cancer, making it the second PD-L1 inhibitor approved for listing in China. Considering that the indication for triple negative breast cancer has been approved in the United States, approval of treatment for TNBC in China may also be a matter of time.

To explore the economic burden induced by the new treatment regimen, we looked up the bidding price of atezolizumab and performed a cost-effectiveness analysis. To our knowledge, this is the first study to evaluate the health and economic outcomes of treatment with atezolizumab 


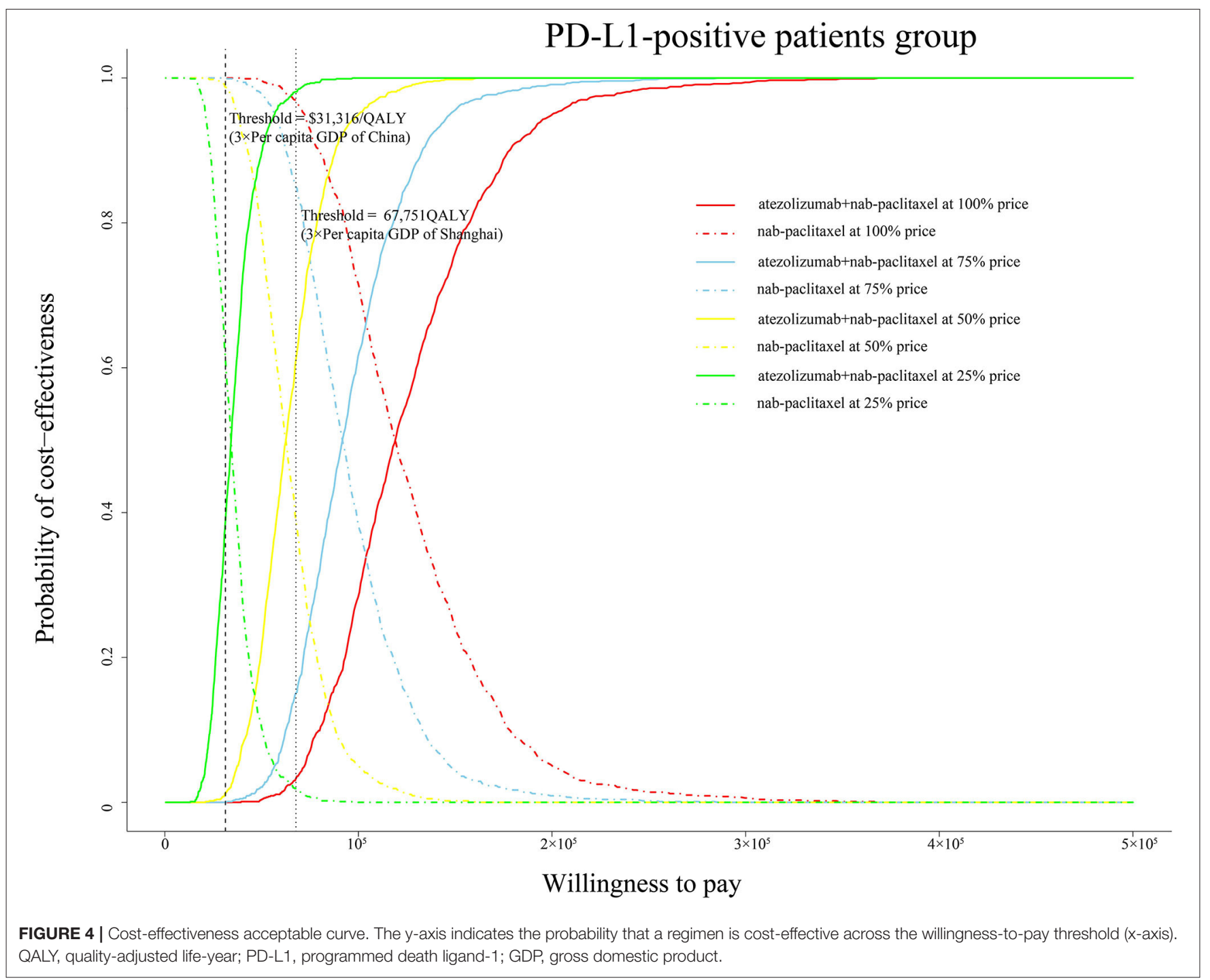

plus nab-paclitaxel vs. paclitaxel regimen in TNBC patients from the perspective of Chinese health sector. Our base-case analysis showed that the ICER of atezolizumab plus nabpaclitaxel vs. nab-paclitaxel in the ITT, PD-L1(+) and PD-L1(-) group is, respectively, \$176,056/QALY, \$118,146/QALY, and $\$ 323,077 / \mathrm{Q} A L Y$. The ICER values exceed the average threshold of $\$ 31,316 / \mathrm{QALY}$ in China. The most obvious finding to emerge from the analysis result is that the PD-L1-positive group gets better advantage of survival. One-way sensitivity analysis showed the range of ICER was from $\$ 89,792 /$ QALY to $\$ 146,514 /$ QALY in the PD-L1-positive group. The lowest value still exceeds the threshold of \$31,316/QALY. Tornado diagram of PD-L1-positive group demonstrated that the price of atezolizumab was the most influential model input. Utility of $\mathrm{PD}$, discount rate and utility of PF were also found to be key factors. Our probabilistic sensitivity analysis additionally simulated various conditions covered the price of atezolizumab reduced by 25,50 , and $75 \%$. If adjust the price of atezolizumab to $25 \%$ in the PD-L1-positive group, the likelihood of atezolizumab plus nab-paclitaxel regimen being cost-effective is $38.7 \%$. In the case of the current threshold, the price needs to be reduced by a large margin. If the threshold of $\$ 67,751 /$ QALY in Shanghai is used as a comparative standard, then the probability of cost-effectiveness is, respectively, 15.1, 61, and $98.1 \%$ at the 75,50 , and $25 \%$ of price of atezolizumab. The results reflect that the price of atezolizumab is too high and the average WTP threshold in China is low, which makes it difficult to change the conclusion that atezolizumab plus nab-paclitaxel regimen is not cost-effective choice.

Currently, we know that some researcher conducted economic evaluations from the point of view of other countries. Wu et al. found that atezolizumab is cost-effective option for PDL1-positive patients with a threshold of $\$ 200,000 /$ QALY in the US (29). However, another US-based study conducted by $\mathrm{Li}$ et al. showed that atezolizumab plus nab-paclitaxel were not considered cost-effective regimen (30). Phua et al. concluded that adding atezolizumab to nab-paclitaxel was not cost-effective for treatment of PD-L1-positive TNBC based on the context of Singapore (31). Although the cost-effectiveness of atezolizumab 
plus chemotherapy based on nab-paclitaxel regimen differs from various views, these evaluations all implied that the price of atezolizumab is too high. With the demand for treatment of TNBC soaring, there is still room for price reduction in the future.

Our study has limitations. First, our survival data derived from IMpassion130, in which the patients were predominantly white. Asian patients accounted for a relatively low proportion. However, our study was made from the perspective of China. Inevitably, the analysis result was slightly affected by race. Second, our study was conducted based on modeling techniques. IPD applied to model was not actual IPD but projected IPD generated according to the specific algorithm. Then, analysis results using parametric model to extrapolate the survival outcomes beyond the time horizon could have a slight hypothesis bias compared with analysis results with sufficient survival data of follow-up. It could undermine the robustness, but our sensitivity analyses covered substantial ranges of all variables. It can well foresee some changes in results induced by modeling techniques. Finally, no research about quality-of-life was conducted in IMpassion 130 trial, direct data sources were lacked. Therefore, the utility data were determined based on published literatures. It would lead to deviations in the cumulative QALYs and may be distinct from actual quality- of-life data. In addition to limitations, we made many efforts in the stage of selecting models. We considered the Markov model, partitioned survival model and the cure model (32). According to the characteristics of the survival curve, we exclude the application of the cure model. Likewise, in order to reduce the deviation caused by the hypothesis, we finally chose the latter between the Markov model and the partitioned survival model. The partitioned survival model could directly obtain survival cohort proportion from survival curve, which can decrease the hypothesis bias of calculating transition probability of PF or PD state to death state.

\section{REFERENCES}

1. Harbeck N, Gnant M. Breast cancer. Lancet Lond Engl. (2017) 389:113450. doi: 10.1016/S0140-6736(16)31891-8

2. Bray F, Ferlay J, Soerjomataram I, Siegel RL, Torre LA, Jemal A. Global cancer statistics 2018: GLOBOCAN estimates of incidence and mortality worldwide for 36 cancers in 185 countries. CA Cancer J Clin. (2018) 68:394424. doi: $10.3322 /$ caac. 21492

3. Rivera-Franco MM, Leon-Rodriguez E. Delays in breast cancer detection and treatment in developing countries. Breast Cancer Basic Clin Res. (2018) 12:1178223417752677. doi: 10.1177/1178223417752677

4. Blows FM, Driver KE, Schmidt MK, Broeks A, van Leeuwen FE, Wesseling J, et al. Subtyping of breast cancer by immunohistochemistry to investigate a relationship between subtype and short and long term survival: a collaborative analysis of data for 10,159 cases from 12 studies. PLoS Med. (2010) 7:e1000279. doi: 10.1371/journal.pmed.1000279

5. Wu Y, Meng Q, Yang Z, Shi L, Hu R, Zhang P, et al. Circulating HER2 mRNA in the peripheral blood as a potential diagnostic and prognostic biomarker in females with breast cancer. Oncol Lett. (2018) 16:372634. doi: 10.3892/ol.2018.9091

6. Valdés-Ferrada J, Muñoz-Durango N, Pérez-Sepulveda A, Muñiz S, Coronado-Arrázola I, Acevedo F, et al. Peripheral blood classical monocytes and plasma interleukin 10 are associated to neoadjuvant

\section{CONCLUSIONS}

In summary, the addition of atezolizumab to nab-paclitaxel could improve the survival time significantly in the PD-L1-positive group, but it is not a cost-effective strategy compared to nabpaclitaxel monotherapy for patients with advanced or metastatic triple-negative breast cancer in the current economic context of China.

\section{DATA AVAILABILITY STATEMENT}

The original contributions presented in the study are included in the article/Supplementary Material, further inquiries can be directed to the corresponding author/s.

\section{ETHICS STATEMENT}

This study was based on a literature review and modeling techniques. This study didn't require approval by an Institutional Research Ethics Board.

\section{AUTHOR CONTRIBUTIONS}

$\mathrm{XL}$ and $\mathrm{YZ}$ was involved in the design of the study. YLa collected the data, performed the economic analysis, and wrote the first draft of the manuscript. YLi collected data and verified results. All authors approved this version for publication.

\section{SUPPLEMENTARY MATERIAL}

The Supplementary Material for this article can be found online at: https://www.frontiersin.org/articles/10.3389/fpubh. 2021.756899/full\#supplementary-material chemotherapy response in breast cancer patients. Front Immunol. (2020) 11:1413. doi: 10.3389/fimmu.2020.01413

7. Abramson VG, Lehmann BD, Ballinger TJ, Pietenpol JA. Subtyping of triple-negative breast cancer: implications for therapy. Cancer. (2015) 121:816. doi: 10.1002/cncr.28914

8. Caparica R, Lambertini M, de Azambuja E. How I treat metastatic triple-negative breast cancer. ESMO Open. (2019) 4:e000504. doi: 10.1136/esmoopen-2019-000504

9. Kumari S, Mohan MG, Shailender G, Badana AK, Malla RR. Synergistic enhancement of apoptosis by coralyne and paclitaxel in combination on MDA-MB-231 a triple-negative breast cancer cell line. J Cell Biochem. (2019) 120:18104-16. doi: 10.1002/jcb.29114

10. Vital D, Ikenberg $K$, Moch $H$, Rössle M, Huber GF. The expression of PD-L1 in salivary gland carcinomas. Sci Rep. (2019) 9:12724. doi: 10.1038/s41598-019-49215-9

11. Choi J, Lee HJ, Yoon S, Ryu H-M, Lee E, Jo Y, et al. Blockade of CCL2 expression overcomes intrinsic PD-1/PD-L1 inhibitor-resistance in transglutaminase 2-induced $\mathrm{PD}$-L1 positive triple negative breast cancer. Am J Cancer Res. (2020) 10:2878-94.

12. Cortes J, Cescon DW, Rugo HS, Nowecki Z, Im S-A, Yusof MM, et al. Pembrolizumab plus chemotherapy versus placebo plus chemotherapy for previously untreated locally recurrent inoperable or metastatic triple-negative breast cancer (KEYNOTE-355): a randomised, 
placebo-controlled, double-blind, phase 3 clinical trial. Lancet. (2020) 396:1817-28. doi: 10.1016/S0140-6736(20)32531-9

13. Schmid P, Adams S, Rugo HS, Schneeweiss A, Barrios $\mathrm{CH}$, Iwata $\mathrm{H}$, et al. Atezolizumab and Nab-paclitaxel in advanced triple-negative breast cancer. N Engl J Med. (2018) 379:2108-21. doi: 10.1056/NEJMoa1809615

14. Schmid P, Rugo HS, Adams S, Schneeweiss A, Barrios $\mathrm{CH}$, Iwata $\mathrm{H}$, et al. Atezolizumab plus nab-paclitaxel as first-line treatment for unresectable, locally advanced or metastatic triple-negative breast cancer (IMpassion130): updated efficacy results from a randomised, doubleblind, placebo-controlled, phase 3 trial. Lancet Oncol. (2020) 21:4459. doi: 10.1016/S1470-2045(19)30689-8

15. Guyot P, Ades A, Ouwens MJ, Welton NJ. Enhanced secondary analysis of survival data: reconstructing the data from published Kaplan-Meier survival curves. BMC Med Res Methodol. (2012) 12:9. doi: 10.1186/1471-2288-12-9

16. Kuk D, Varadhan R. Model selection in competing risks regression. Stat Med. (2013) 32:3077-88. doi: 10.1002/sim.5762

17. Lu S, Ye M, Ding L, Tan F, Fu J, Wu B. Cost-effectiveness of gefitinib, icotinib, and pemetrexed-based chemotherapy as first-line treatments for advanced non-small cell lung cancer in China. Oncotarget. (2017) 8:999610006. doi: 10.18632/oncotarget. 14310

18. Chinese Drug Price of Drug Centralized Bid Procurement (2021). Available online at: https://db.yaozh.com/yaopinzhongbiao (accessed January 20, 2021).

19. Rui M, Shi F, Shang Y, Meng R, Li H. Economic evaluation of cisplatin plus gemcitabine versus paclitaxel plus gemcitabine for the treatment of first-line advanced metastatic triple-negative breast cancer in China: using Markov model and partitioned survival model. Adv Ther. (2020) 37:376174. doi: 10.1007/s12325-020-01418-7

20. Wu B, Zhang Q, Sun J. Cost-effectiveness of nivolumab plus ipilimumab as first-line therapy in advanced renal-cell carcinoma. I Immunother Cancer. (2018) 6:124. doi: 10.1186/s40425-018-0440-9

21. Lang Y, Dong D. Cetuximab plus chemotherapy versus chemotherapy alone in recurrent or metastatic head and neck squamous cell carcinoma: a cost-effectiveness analysis. Cancer Manag Res. (2020) 12:11383-90. doi: 10.2147/CMAR.S272149

22. Lang $\mathrm{Y}$, Dong $\mathrm{D}, \mathrm{Wu}$ B. Pembrolizumab vs the EXTREME regimen in recurrent or metastatic head and neck squamous cell carcinoma: a cost-effectiveness analysis. Clin Drug Investig. (2020) 40:1137-46. doi: 10.1007/s40261-020-00973-9

23. Paracha N, Thuresson P-O, Moreno SG, MacGilchrist KS. Health state utility values in locally advanced and metastatic breast cancer by treatment line: a systematic review. Expert Rev Pharmacoecon Outcomes Res. (2016) 16:54959. doi: 10.1080/14737167.2016.1222907

24. Mistry R, May JR, Suri G, Young K, Brixner D, Oderda G, et al. Costeffectiveness of ribociclib plus letrozole versus palbociclib plus letrozole and letrozole monotherapy in the first-line treatment of postmenopausal women with HR+/HER2- advanced or metastatic breast cancer: A U.S. payer perspective. J Manag Care Spec Pharm. (2018) 24:51423. doi: 10.18553/jmcp.2018.24.6.514
25. Zhao $\mathrm{Y}$, Feng $\mathrm{H}, \mathrm{Qu}$ J, Luo $\mathrm{X}$, Ma W, Tian J. A systematic review of pharmacoeconomic guidelines. J Med Econ. (2018) 21:85-96. doi: 10.1080/13696998.2017.1387118

26. Hutubessy R, Chisholm D, Edejer TT-T. Generalized costeffectiveness analysis for national-level priority-setting in the health sector. Cost Eff Resour Alloc CE. (2003) 1:8. doi: 10.1186/1478-75 47-1-8

27. Briggs AH, Weinstein MC, Fenwick EAL, Karnon J, Sculpher MJ, Paltiel $\mathrm{AD}$, et al. Model parameter estimation and uncertainty analysis: a report of the ISPOR-SMDM modeling good research practices task force working group-6. Med Decis Mak Int J Soc Med Decis Mak. (2012) 32:72232. doi: $10.1177 / 0272989 X 12458348$

28. Klinke DJ. Is immune checkpoint modulation a potential therapeutic option in triple negative breast cancer? Breast Cancer Res. (2014) 16:457. doi: 10.1186/s13058-014-0457-z

29. $\mathrm{Wu} \mathrm{B}, \mathrm{Ma}$ F. Cost-effectiveness of adding atezolizumab to first-line chemotherapy in patients with advanced triple-negative breast cancer. Ther Adv Med Oncol. (2020) 12:175883592091600. doi: 10.1177/17588359209 16000

30. Li J, Zhang T, Lu P, Zhao J, Chen L, Jiang J. Cost-effectiveness analysis of atezolizumab plus nab-paclitaxel for untreated metastatic triple-negative breast cancer. Immunotherapy. (2020) 12:705-13. doi: 10.2217/imt-2020-0036

31. Phua LC, Lee SC, Ng K, Abdul Aziz MI. Cost-effectiveness analysis of atezolizumab in advanced triple-negative breast cancer. BMC Health Serv Res. (2020) 20:581. doi: 10.1186/s12913-020-05445-6

32. Woods B, Sideris E, Palmer S, Latimer N, Soares M. Nice Dsu Technical Support Document 19: Partitioned Survival Analysis for Decision Modelling in Health Care: A Critical Review. (2021). Available online at: http://nicedsu. org.uk/technical-support-documents/partitioned-survival-analysis-tsd/ (accessed February 11, 2021).

Conflict of Interest: The authors declare that the research was conducted in the absence of any commercial or financial relationships that could be construed as a potential conflict of interest.

Publisher's Note: All claims expressed in this article are solely those of the authors and do not necessarily represent those of their affiliated organizations, or those of the publisher, the editors and the reviewers. Any product that may be evaluated in this article, or claim that may be made by its manufacturer, is not guaranteed or endorsed by the publisher.

Copyright (C) 2021 Liu, Lang, Liao and Zhu. This is an open-access article distributed under the terms of the Creative Commons Attribution License (CC BY). The use, distribution or reproduction in other forums is permitted, provided the original author(s) and the copyright owner(s) are credited and that the original publication in this journal is cited, in accordance with accepted academic practice. No use, distribution or reproduction is permitted which does not comply with these terms. 